\title{
Cullis-Radić determinant of a rectangular matrix which has a number of identical columns
}

\begin{abstract}
In this paper we present how identical columns affect the CullisRadić determinant of an $m \times n$ matrix, where $m \leq n$.
\end{abstract}

1. Introduction. In 1913, Cullis [4] introduced the concept of determinoid of a rectangular matrix and it is probably the first published generalization of the determinant of a square matrix. Cullis reserved the name determinant for square matrices only, but nowadays this term is also used for rectangular matrices.

Since the original Cullis's definition of the determinant (determinoid) of a rectangular matrix is descriptive and requires some nonstandard terminology to be introduced first, we reformulate the idea of Cullis in the following way:

Definition 1.1 (Cullis [4, $§ 3]$, reformulated). Let $A=\left\{a_{i j}\right\}$ be an $m \times n$ matrix with $m$ rows and $n$ columns, with elements $a_{i j}$, where $1 \leq i \leq m$, $1 \leq j \leq n$, and let $k=\min \{m, n\}$. The determinant of $A$ is defined as the sum

$$
\operatorname{det} A=\sum_{P}(-1)^{\alpha(P)} P
$$

2010 Mathematics Subject Classification. 15A15.

Key words and phrases. Determinant, rectangular matrix, Cullis-Radić determinant, repeated columns. 
over all products $P=a_{i_{1} j_{1}} \ldots a_{i_{k} j_{k}}$ of elements of $A$ taken from different rows and different columns with

$$
\alpha(P)=\sum_{p=1}^{k}\left[\left(i_{p}-\sum_{\substack{1 \leq q \leq p \\ i_{q} \leq i_{p}}} 1\right)+\left(j_{p}-\sum_{\substack{1 \leq q \leq p \\ j_{q} \leq j_{p}}} 1\right)\right]
$$

where the expression in square brackets is equal to the sum of horizontal and vertical steps one must take to pass from the element $a_{i_{p} j_{p}}$ to the element $a_{11}$ in the matrix obtained from $A$ by removing rows and columns containing the elements $a_{i_{q} j_{q}}$ for $q<p$.

Independently of Cullis, in 1966, Radić [11] proposed the following definition of the determinant, which turns out to be equivalent to the Cullis's definition (the equivalence follows from $[4, \S 30]$ ).

Definition 1.2 (Radić [11, Definition 1]). Let $A=\left[A_{1}, \ldots, A_{n}\right]$ be an $m \times n$ matrix with $n$ columns $A_{1}, \ldots, A_{n}$ and $m \leq n$. The determinant of $A$ is defined as the sum

$$
\operatorname{det} A=\sum_{1 \leq j_{1}<\ldots<j_{m} \leq n}(-1)^{r+j_{1}+\ldots+j_{m}} \operatorname{det}\left[A_{j_{1}}, \ldots, A_{j_{m}}\right],
$$

where $r=1+2+\ldots+m$. If $m>n$, then $\operatorname{det} A=\operatorname{det} A^{T}$.

It is worth noting here that there are also other definitions of the determinant of a rectangular matrix which are not equivalent to the definitions of Cullis and Radić, see for example [2, 3, 5, 9, 18, 19, 21].

The Cullis-Radic determinant of an $m \times n$ matrix, where $m \leq n$, and the classical determinant of a square matrix have several common properties, see $[4, \S 5, \S 27, \S 32]$ and $[11,16]$, for example:

(1) The Cullis-Radić determinant of a matrix is a linear function of its rows.

(2) If a matrix $A$ has two identical rows or one of its rows is a linear combination of other rows, then the Cullis-Radić determinant of $A$ is equal to zero.

(3) Interchanging any two rows of a matrix changes the sign of its CullisRadić determinant.

(4) Adding a linear combination of rows to another row does not change the Cullis-Radić determinant.

(5) The Cullis-Radić determinant can be calculated using the Laplace expansion with respect to a row.

More algebraic properties, which characterize the Cullis-Radić determinant, can be found in $[1,4,6,8,12,13,14,16,20]$, and some geometric interpretations are presented in $[7,10,12,13,14,15,17,20]$.

In this paper, we are going to present how identical columns affect the Cullis-Radić determinant of an $m \times n$ matrix, where $m \leq n$. The rest 
of the paper is organized as follows. In Section 2, we consider the CullisRadić determinant of matrices with two identical columns. In Section 3 and Section 4, we present the results for matrices which have an arbitrary number of identical adjacent columns and an arbitrary number of identical adjacent pairs of columns. Matrices formed by identical adjacent sequences of columns are the subject of Section 5 .

2. Two identical columns. First, we introduce some useful notation and recall from [6] how interchanging two columns affects the determinant of a rectangular matrix.

Let $A=\left[A_{1}, \ldots, A_{n}\right]$ be an $m \times n$ matrix, where $m \leq n$. We use the following notation:

(i) $A_{i \leftrightarrow j}$, where $i, j \in\{1,2, \ldots, n\}, i \neq j$, denotes the matrix obtained from $A$ by interchanging columns $A_{i}$ and $A_{j}$,

(ii) $D_{i}(A)=\left[A_{1}, \ldots, A_{i-1}, A_{i+1}, \ldots, A_{n}\right]$, where $i \in\{1,2, \ldots, n\}$ and $n>1$, denotes the matrix obtained from $A$ by removing the column $A_{i}$,

(iii) $I_{i}^{K}(A)=\left[A_{1}, \ldots, A_{i-1}, K, A_{i}, A_{i+1}, \ldots, A_{n}\right]$, where $i \in\{1,2, \ldots, n\}$, denotes the matrix obtained from $A$ by inserting the column $K$ before the $i$-th column of $A$.

Lemma 2.1 ([6, Theorem 2.6]). Let $A$ be an $m \times(m+1)$ matrix. Then for all $i, j \in\{1,2, \ldots, m+1\}$ such that $i \neq j$, we have

$$
\operatorname{det} A+\operatorname{det} A_{i \leftrightarrow j}=0 .
$$

Lemma 2.2 ([6, Theorem 2.7]). Let $A=\left[A_{1}, \ldots, A_{n}\right]$ be an $m \times n$ matrix, where $m \leq n$. Then

$$
\begin{aligned}
& \operatorname{det} A+\operatorname{det} A_{i \leftrightarrow j}= 2 \sum_{\substack{1 \leq j_{1}<\ldots<j_{m} \leq n \\
i, j \notin\left\{j_{1}, \ldots, j_{m}\right\}}}(-1)^{r+j_{1}+j_{2}+\ldots+j_{m}} \operatorname{det}\left[A_{j_{1}}, A_{j_{2}}, \ldots, A_{j_{m}}\right] \\
&+2 \sum_{\substack{1 \leq j_{1}<\ldots<j_{m} \leq n \\
(i \in J, j \notin J \text { or } i \notin J, j \in J)}}(-1)^{r+j_{1}+j_{2}+\ldots+j_{m}} \operatorname{det}\left[A_{j_{1}}, A_{j_{2}}, \ldots, A_{j_{m}}\right], \\
& J=\{\min \{i, j\}, \ldots, \max \{i, j\}\} \backslash\left\{j_{1}, \ldots, j_{m}\right\} \\
& \operatorname{card}(J) \equiv 0(\bmod 2)
\end{aligned}
$$

where $r=1+2+\ldots+m$ and $\operatorname{card}(X)$ stands for the cardinality of $X$.

Corollary 2.3 ([6, Corollary 2.8]). Let $A$ be an $m \times n$ matrix, where $m \leq n$. Then for every $i \in\{1,2, \ldots, n-1\}$, we have

$$
\operatorname{det} A+\operatorname{det} A_{i \leftrightarrow(i+1)}= \begin{cases}0, & \text { if } n \in\{m, m+1\}, \\ 2 \operatorname{det} D_{i}\left(D_{i+1}(A)\right), & \text { if } n \geq m+2 .\end{cases}
$$


The following theorem gives a sufficient condition for a matrix to have the determinant equal to zero and shows that the determinant of a matrix which has two identical columns can be expressed by determinants of matrices obtained from the given matrix by removing one or both identical columns.

Theorem 2.4. Let $A=\left[A_{1}, \ldots, A_{n}\right]$ be an $m \times n$ matrix, where $m \leq n$ and $A_{i}=A_{j}$ for some $i, j \in\{1,2, \ldots, n\}$ such that $i<j$. Then

$$
\operatorname{det} A= \begin{cases}0, & \text { if } n \in\{m, m+1\}, \\ \operatorname{det} D_{i}\left(D_{j}(A)\right), & \text { if } n \geq m+2 \text { and } j=i+1, \\ 2 \sum_{p=i+1}^{j-1}(-1)^{p-i-1} \operatorname{det} D_{i}\left(D_{p}(A)\right) & \\ \quad+(-1)^{j-i-1} \operatorname{det} D_{i}\left(D_{j}(A)\right), & \text { if } n \geq m+2 \text { and } j>i+1 .\end{cases}
$$

Proof. If $n=m+1$, then we apply Lemma 2.1 and obtain

$$
2 \operatorname{det} A=\operatorname{det} A+\operatorname{det} A_{i \leftrightarrow j}=0 .
$$

In the case where $n \geq m+2$ and $j=i+1$, by Corollary 2.3, we have

$$
2 \operatorname{det} A=\operatorname{det} A+\operatorname{det} A_{i \leftrightarrow j}=2 \operatorname{det} D_{i}\left(D_{j}(A)\right) \text {. }
$$

If $n \geq m+2$ and $j>i+1$, then for every $k \in\{0,1, \ldots, j-i-2\}$ Corollary 2.3 yields

$$
\operatorname{det} I_{i+k}^{A_{i}}\left(D_{i}(A)\right)=2 \operatorname{det} D_{i}\left(D_{i+k+1}(A)\right)-\operatorname{det} I_{i+k+1}^{A_{i}}\left(D_{i}(A)\right) .
$$

From the previous case it follows that

$$
\operatorname{det} I_{j-1}^{A_{i}}\left(D_{i}(A)\right)=\operatorname{det} D_{i}\left(D_{j}(A)\right) .
$$

Therefore, using (2.1), we obtain

$$
\begin{aligned}
\operatorname{det} A & =\operatorname{det} I_{i}^{A_{i}}\left(D_{i}(A)\right)=2 \operatorname{det} D_{i}\left(D_{i+1}(A)\right)-\operatorname{det} I_{i+1}^{A_{i}}\left(D_{i}(A)\right) \\
& =2 \operatorname{det} D_{i}\left(D_{i+1}(A)\right)-2 \operatorname{det} D_{i}\left(D_{i+2}(A)\right)+\operatorname{det} I_{i+2}^{A_{i}}\left(D_{i}(A)\right) \\
& =2 \sum_{p=i+1}^{j-1}(-1)^{p-i-1} \operatorname{det} D_{i}\left(D_{p}(A)\right)+(-1)^{j-i-1} \operatorname{det} D_{i}\left(D_{j}(A)\right) .
\end{aligned}
$$

Remark 2.5. In the proof of Theorem 2.4, instead of (2.1) the following relation:

$$
\operatorname{det} I_{j-k}^{A_{j}}\left(D_{j}(A)\right)=2 \operatorname{det} D_{j}\left(D_{j-k-1}(A)\right)-\operatorname{det} I_{j-k-1}^{A_{j}}\left(D_{j}(A)\right)
$$

for $k \in\{0,1, \ldots, j-i-2\}$ can also be used, yielding

$$
\operatorname{det} A=2 \sum_{p=i+1}^{j-1}(-1)^{j-p-1} \operatorname{det} D_{j}\left(D_{p}(A)\right)+(-1)^{j-i-1} \operatorname{det} D_{j}\left(D_{i}(A)\right)
$$

for $n \geq m+2$ and $j>i+1$. 
Example 2.6. Let $A=\left[A_{1}, A_{2}, A_{3}, A_{4}, A_{5}, A_{6}\right]$ be a $3 \times 6$ matrix.

(a) If $A_{2}=A_{5}$, then

$$
\begin{aligned}
\operatorname{det} A & =2 \operatorname{det} D_{2}\left(D_{3}(A)\right)-2 \operatorname{det} D_{2}\left(D_{4}(A)\right)+\operatorname{det} D_{2}\left(D_{5}(A)\right) \\
& =2 \operatorname{det}\left[A_{1}, A_{4}, A_{5}, A_{6}\right]-2 \operatorname{det}\left[A_{1}, A_{3}, A_{5}, A_{6}\right]+\operatorname{det}\left[A_{1}, A_{3}, A_{4}, A_{6}\right]
\end{aligned}
$$

and

$$
\begin{aligned}
\operatorname{det} A & =2 \operatorname{det} D_{5}\left(D_{4}(A)\right)-2 \operatorname{det} D_{5}\left(D_{3}(A)\right)+\operatorname{det} D_{5}\left(D_{2}(A)\right) \\
& =2 \operatorname{det}\left[A_{1}, A_{2}, A_{3}, A_{6}\right]-2 \operatorname{det}\left[A_{1}, A_{2}, A_{4}, A_{6}\right]+\operatorname{det}\left[A_{1}, A_{3}, A_{4}, A_{6}\right] .
\end{aligned}
$$

(b) If $A_{2}=A_{3}$, then

$$
\operatorname{det} A=\operatorname{det}\left[A_{1}, A_{4}, A_{5}, A_{6}\right] \text {. }
$$

3. An arbitrary number of identical adjacent columns. In this section, we present two theorems which follow easily from the fact that, while calculating the determinant of an $m \times n$ matrix, two adjacent identical columns can be canceled if $n \geq m+2$ (see Theorem 2.4).

Theorem 3.1. Let $A=\left[A_{1}, \ldots, A_{n}\right]$ be an $m \times n$ matrix, where $m \leq n$. Fix $i \in\{1,2, \ldots, n\}$ and replace the column $A_{i}$ in the matrix $A$ with $k$ copies of $A_{i}$, where $k \geq 2$, obtaining in this way a matrix $B$ of the form

$$
B=[A_{1}, \ldots, A_{i-1}, \underbrace{A_{i}, \ldots, A_{i}}_{k \text { columns }}, A_{i+1}, \ldots, A_{n}] .
$$

Then

$$
\operatorname{det} B= \begin{cases}\operatorname{det} A, & \text { if } k \text { is odd, } \\ 0, & \text { if } k \text { is even and } m=n, \\ \operatorname{det} D_{i}(A), & \text { if } k \text { is even and } m<n .\end{cases}
$$

Theorem 3.2. Let $A=\left[A_{1}, \ldots, A_{n}\right]$ be an $m \times n$ matrix, where $m \leq n$. For each $i \in\{1,2, \ldots, n\}$ replace the column $A_{i}$ in the matrix $A$ with $k_{i}$ copies of $A_{i}$, where $k_{i} \geq 1$, obtaining in this way a matrix $B$ of the form

$$
B=[\underbrace{A_{1}, \ldots, A_{1}}_{k_{1} \text { columns }}, \underbrace{A_{2}, \ldots, A_{2}}_{k_{2} \text { columns }}, \ldots, \underbrace{A_{n}, \ldots, A_{n}}_{k_{n} \text { columns }}] \text {. }
$$

If $p$ is the number of all odd integers among $k_{1}, \ldots, k_{n}$ and $\left(k_{j_{1}}, \ldots, k_{j_{p}}\right)$ is the subsequence of all odd numbers taken from the sequence $\left(k_{1}, \ldots, k_{n}\right)$, then

$$
\operatorname{det} B= \begin{cases}\operatorname{det} A, & \text { if } p=n\left(\text { all } k_{i} \text { 's are odd }\right), \\ \operatorname{det}\left[A_{j_{1}}, \ldots, A_{j_{p}}\right], & \text { if } m \leq p<n, \\ 0, & \text { if } p<m .\end{cases}
$$

Example 3.3. If $A=\left[A_{1}, A_{2}, A_{2}, A_{2}, A_{3}, A_{4}\right]$ is a $2 \times 6$ matrix and

$$
B=\left[B_{1}, B_{2}, B_{2}, B_{3}, B_{3}, B_{3}, B_{4}, B_{5}, B_{5}, B_{5}, B_{5}, B_{6}\right]
$$

is a $3 \times 12$ matrix, then

$$
\operatorname{det} A=\operatorname{det}\left[A_{1}, A_{2}, A_{3}, A_{4}\right] \text { and } \operatorname{det} B=\operatorname{det}\left[B_{1}, B_{3}, B_{4}, B_{6}\right] .
$$




\section{An arbitrary number of identical adjacent pairs of columns.}

Theorem 4.1. Let $A=\left[A_{1}, \ldots, A_{n}\right]$ be an $m \times n$ matrix, where $m \leq n$. Fix $i \in\{1,2, \ldots, m-1\}$ and replace the pair of columns $A_{i}, A_{i+1}$ in the matrix $A$ with $k$ copies of the pair $A_{i}, A_{i+1}$, where $k \geq 2$, obtaining in this way a matrix $B_{k}$ of the form

$$
B_{k}=[A_{1}, \ldots, A_{i-1}, \underbrace{A_{i}, A_{i+1}, \ldots, A_{i}, A_{i+1}}_{k \text { pairs } A_{i} A_{i+1}}, A_{i+2}, \ldots, A_{n}] .
$$

Then

(4.1) $\operatorname{det} B_{k}= \begin{cases}k \operatorname{det} A, & \text { if } n \in\{m, m+1\}, \\ k \operatorname{det} A-(k-1) \operatorname{det} D_{i}\left(D_{i+1}(A)\right), & \text { if } n \geq m+2 .\end{cases}$

Proof. First, we prove by induction that for every positive integer $k$

$$
\operatorname{det} B_{k}=(k-1) \operatorname{det} B_{2}-(k-2) \operatorname{det} A \text {. }
$$

The cases $k=1$ and $k=2$ are easy. For $k \geq 3$, applying Corollary 2.3, we have

$$
\begin{aligned}
\operatorname{det} B_{k}+ & \operatorname{det}[A_{1}, \ldots, A_{i-1}, A_{i+1}, A_{i}, \underbrace{A_{i}, A_{i+1}, \ldots, A_{i}, A_{i+1}}_{k-1 \text { pairs } A_{i} A_{i+1}}, A_{i+2}, \ldots, A_{n}] \\
& =2 \operatorname{det} B_{k-1} .
\end{aligned}
$$

Theorem 3.1 yields

$$
\operatorname{det} B_{k}+\operatorname{det} B_{k-2}=2 \operatorname{det} B_{k-1} .
$$

Assuming that

$$
\begin{aligned}
& \operatorname{det} B_{k-1}=(k-2) \operatorname{det} B_{2}-(k-3) \operatorname{det} A, \\
& \operatorname{det} B_{k-2}=(k-3) \operatorname{det} B_{2}-(k-4) \operatorname{det} A,
\end{aligned}
$$

we obtain

$$
\operatorname{det} B_{k}=2 \operatorname{det} B_{k-1}-\operatorname{det} B_{k-2}=(k-1) \operatorname{det} B_{2}-(k-2) \operatorname{det} A .
$$

Considering (4.3) for $k=2$, and then applying Theorem 2.4, we have

$$
\operatorname{det} B_{2}= \begin{cases}2 \operatorname{det} A, & \text { if } n \in\{m, m+1\}, \\ 2 \operatorname{det} A-\operatorname{det} D_{i}\left(D_{i+1}(A)\right), & \text { if } n \geq m+2 .\end{cases}
$$

Now, combining (4.2) and (4.4), we obtain (4.1).

Example 4.2. Let

$$
A=\left[A_{1}, A_{2}, A_{3}, A_{4}\right] \text { and } B=\left[A_{1}, A_{2}, A_{1}, A_{2}, A_{1}, A_{2}, A_{3}, A_{4}\right] .
$$

(a) If $A$ is a $2 \times 4$ matrix and $B$ is a $2 \times 8$ matrix, then

$$
\operatorname{det} B=3 \operatorname{det} A-2 \operatorname{det}\left[A_{3}, A_{4}\right] \text {. }
$$

(b) If $A$ is a $3 \times 4$ matrix and $B$ is a $3 \times 8$ matrix, then

$$
\operatorname{det} B=3 \operatorname{det} A \text {. }
$$




\section{An arbitrary number of identical adjacent sequences of columns} which form the entire matrix. In this section we prove the following theorem.

Theorem 5.1. Let $k$ be a positive integer. If $A$ is an $m \times n$ matrix, where $m \leq n$, then

$$
\operatorname{det}[\underbrace{A, A, \ldots, A}_{k \text { copies of } A}]= \begin{cases}k^{m / 2} \operatorname{det} A, & \text { if } m \text { is even, } \\ k^{(m+1) / 2} \operatorname{det} A, & \text { if } m \text { is odd and } n \text { is even, } \\ 0, & \text { if } m, n \text { are odd and } k \text { is even, } \\ k^{(m-1) / 2} \operatorname{det} A, & \text { if } m, n, k \text { are odd. }\end{cases}
$$

Proof. The proof follows immediately from Lemma 5.2, Lemma 5.3 and Lemma 5.7, which are proved below.

Before we start with the first lemma, we introduce some useful notation. Assume that

$$
A=\left[A_{1}, \ldots, A_{n}\right]
$$

is an $m \times n$ matrix with $n$ columns $A_{1}, \ldots, A_{n}, m \leq n$, and

$$
B=[\underbrace{A, A, \ldots, A}_{k \text { copies of } A}]=\left[\mathcal{B}_{1}, \ldots, \mathcal{B}_{k}\right]=\left[B_{1}, \ldots, B_{n k}\right]
$$

is an $m \times n k$ matrix, where $\mathcal{B}_{i}=A$ for every $i=1,2, \ldots, k$, and $B_{1}, \ldots, B_{n k}$ are the columns of $B$.

For every sequence $a=\left(a_{1}, \ldots, a_{k}\right)$ of nonnegative integers such that $\sum_{i=1}^{k} a_{i}=m$, define $G_{a}(A)$ to be the set of $m \times m$ matrices which have $a_{1}$ columns taken from the matrix $\mathcal{B}_{1}$, followed by $a_{2}$ columns taken from the matrix $\mathcal{B}_{2}$, etc., followed by $a_{k}$ columns taken from the matrix $\mathcal{B}_{k}$-in each group of $a_{i}$ columns, the columns are arranged in the same order as they were arranged in the matrix $\mathcal{B}_{i}, i=1,2, \ldots, n$.

Moreover, for every matrix $M=\left[M_{1}, \ldots, M_{m}\right] \in G_{a}(A)$ such that $M_{i}=$ $B_{j_{i}}$ for each $i=1,2, \ldots, m$, define

$$
c(a, M)=\sum_{i=1}^{m} j_{i} .
$$

Lemma 5.2. Let $A=\left[A_{1}, \ldots, A_{n}\right]$ be an $m \times n$ matrix with $n$ columns $A_{1}, \ldots, A_{n}$, where $m \leq n$, and let $B=\left[\mathcal{B}_{1}, \ldots, \mathcal{B}_{k}\right]$ be an $m \times n k$ matrix, where $\mathcal{B}_{i}=A$ for each $i=1,2, \ldots, k$. Then

$$
\operatorname{det} B=\sum_{\substack{a=\left(a_{1}, \ldots, a_{k}\right) \\ a_{1}+\ldots+a_{k}=m \\ a_{i} \in \mathbb{Z}, a_{i} \geq 0, i=1,2, \ldots, k}} \sum_{M \in G_{a}(A)}(-1)^{r+c(a, M)} \operatorname{det} M,
$$

where $r=1+2+\ldots+m$.

Proof. The proof follows easily from Definition 1.2. 
Let $n, m, k$ be positive integers and $\widetilde{n}=\frac{1-(-1)^{n}}{2} \in\{0,1\}$. For any sequence $\left(a_{1}, \ldots, a_{k}\right)$ of nonnegative integers satisfying $\sum_{i=1}^{k} a_{i}=m$, define $S_{\left(a_{1}, \ldots, a_{k}\right)}^{\widetilde{n}}$ by the following formulas:

$$
\begin{aligned}
& S_{(m)}^{\widetilde{n}}=1, \\
& S_{(m, 0)}^{\widetilde{n}}=1, \\
& S_{(0, m)}^{\widetilde{n}}=(-1)^{m n}, \\
& S_{(1, m-1)}^{\widetilde{n}}= \begin{cases}0, & \text { if } m \text { is even, } \\
1, & \text { if } m \text { is odd, }\end{cases} \\
& S_{(m-1,1)}^{\widetilde{n}}= \begin{cases}0, & \text { if } m \text { is even, } \\
(-1)^{n}, & \text { if } m \text { is odd, }\end{cases} \\
& S_{(u, v)}^{\widetilde{n}}=\sum_{i=0}^{v}(-1)^{i(u+n)} S_{(u-1, v-i)}^{\widetilde{n}},
\end{aligned}
$$

where $u \geq 2, v \geq 2$ are integers and $u+v=m$, $S_{\left(a_{1}, \ldots, a_{p}, 0, \ldots, 0\right)}^{\widetilde{n}}=S_{\left(a_{1}, \ldots, a_{p}\right)}^{\widetilde{n}}$, where $p<k, a_{1} \neq 0, a_{p} \neq 0, k \geq 3$, $S_{\left(a_{1}, \ldots, a_{k}\right)}^{\widetilde{n}}=(-1)^{m n p} S_{\left(a_{p+1}, \ldots, a_{k}\right)}^{\widetilde{n}}$, where $p<k, a_{1}=\ldots=a_{p}=0, a_{p+1} \neq 0, k \geq 3$, $S_{\left(a_{1}, \ldots, a_{k-2}, a_{k-1}, a_{k}\right)}^{\widetilde{n}}=S_{\left(a_{1}, \ldots, a_{k-2}, a_{k-1}+a_{k}\right)}^{\widetilde{\widetilde{n}}} S_{\left(a_{k-1}, a_{k}\right)}^{\widetilde{n}}$, where $a_{1} \neq 0, a_{k} \neq 0, k \geq 3$.

Lemma 5.3. Let $A=\left[A_{1}, \ldots, A_{n}\right]$ be an $m \times n$ matrix with $n$ columns $A_{1}, \ldots, A_{n}$, where $m \leq n$, and let $B=\left[\mathcal{B}_{1}, \ldots, \mathcal{B}_{k}\right]$ be an $m \times n k$ matrix, where $\mathcal{B}_{i}=A$ for each $i=1,2, \ldots, k$. For every sequence of nonnegative integers $a=\left(a_{1}, \ldots, a_{k}\right)$ such that $\sum_{i=1}^{k} a_{i}=m$, we have

$$
\sum_{M \in G_{a}(A)}(-1)^{r+c(a, M)} \operatorname{det} M=S_{a}^{\widetilde{n}} \operatorname{det} A,
$$

where $r=1+\ldots+m$ and $\widetilde{n}=\frac{1-(-1)^{n}}{2}$.

Proof. The proof is done by induction on $k$ and it is divided into nine steps. The first five steps (a)-(e) cover the base cases. The remaining steps (f)-(i) complete the induction.

(a) For $k=1$, we have $B=A$ and (5.2) follows immediately from Lemma 5.2 and formula (5.1a).

(b) For $k=2$ and $a=(m, 0)$, we have $G_{a}(A)=G_{(m, 0)}(A)=G_{(m)}(A)$ and $S_{a}^{\widetilde{n}}=S_{(m, 0)}^{\widetilde{n}}=S_{(m)}^{\widetilde{n}}$. Therefore, applying case (a), we obtain (5.2). 
(c) For $k=2$ and $a=(0, m)$, for every matrix $M=\left[M_{1}, \ldots, M_{m}\right] \in$ $G_{a}(A)$ such that $M_{i}=B_{j_{i}}$ for each $i=1,2, \ldots, m$, we have $M \in G_{(m)}(A)$ and

$$
c(a, M)=\sum_{i=1}^{m} j_{i}=\sum_{i=1}^{m}\left(j_{i}-n\right)+m n=c((m), M)+m n .
$$

Since there is a one-to-one correspondence between matrices in $G_{a}(A)$ and $G_{(m)}(A)$, we have

$$
\begin{aligned}
\sum_{M \in G_{a}(A)}(-1)^{r+c(a, M)} \operatorname{det} M & =(-1)^{m n} \sum_{M \in G_{(m)}(A)}(-1)^{r+c((m), M)} \operatorname{det} M \\
& =(-1)^{m n} \operatorname{det} A .
\end{aligned}
$$

Now, applying (5.1c), we obtain (5.2).

(d) For $k=2$ and $a=(1, m-1)$, every matrix $M \in G_{a}(A)$ can be represented in the form $M=\left[A_{p_{1}}, \ldots, A_{p_{m}}\right]$, where $\left(p_{2}, \ldots, p_{m}\right)$ is an increasing sequence of integers and $p_{i} \in\{1,2, \ldots, n\}, i=1,2, \ldots, m$. Moreover, for every sequence $\left(p_{1}, \ldots, p_{m}\right)$ of integers such that $\left(p_{2}, \ldots, p_{m}\right)$ is increasing and $p_{i} \in\{1,2, \ldots, n\}, i=1,2, \ldots, k$, the matrix $\left[A_{p_{1}}, \ldots, A_{p_{m}}\right]$ is an element of $G_{a}(A)$.

Fix a nondecreasing sequence $\left(p_{1}, \ldots, p_{m}\right)$ of integers from $\{1,2, \ldots, n\}$ and consider the sum

$$
\mathcal{S}\left(p_{1}, \ldots, p_{m}\right)=\sum(-1)^{r+c(a, M)} \operatorname{det} M
$$

over all matrices $M \in G_{a}(A)$ such that $A_{p_{1}}, \ldots, A_{p_{m}}$ are the columns of $M$. Since any of the columns $A_{p_{1}}, \ldots, A_{p_{m}}$ can be the first column of $M$, we have

$$
\begin{aligned}
\mathcal{S}\left(p_{1}, \ldots,\right. & \left.p_{m}\right)=(-1)^{r+p_{1}+\sum_{i=2}^{m}\left(p_{i}+n\right)} \operatorname{det}\left[A_{p_{1}}, A_{p_{2}}, \ldots, A_{p_{m}}\right] \\
& +\sum_{j=2}^{m}(-1)^{r+p_{j}+\sum_{\substack{i=1 \\
i \neq j}}^{m}\left(p_{i}+n\right)} \operatorname{det}\left[A_{p_{j}}, A_{p_{1}}, \ldots, A_{p_{j-1}}, A_{p_{j+1}}, \ldots, A_{p_{m}}\right] \\
= & \begin{cases}(-1)^{r+\sum_{i=1}^{m} p_{i}} \operatorname{det}\left[A_{p_{1}}, \ldots, A_{p_{m}}\right], & \text { if } m \text { is odd and } p_{1}<\ldots<p_{m}, \\
0, & \text { otherwise. }\end{cases}
\end{aligned}
$$

Therefore

$\sum_{M \in G_{a}(A)}(-1)^{r+c(a, M)} \operatorname{det} M=\sum_{p_{1}<\ldots<p_{m}} \mathcal{S}\left(p_{1}, \ldots, p_{m}\right)= \begin{cases}\operatorname{det} A, & \text { if } m \text { is odd } \\ 0, & \text { if } m \text { is even }\end{cases}$ and now (5.2) follows easily from (5.1d). 
(e) For $k=2$ and $a=(m-1,1)$, the proof is similar to the previous case. Fix a nondecreasing sequence $\left(p_{1}, \ldots, p_{m}\right)$ of integers from $\{1,2, \ldots, n\}$ and consider the sum

$$
\mathcal{S}\left(p_{1}, \ldots, p_{m}\right)=\sum(-1)^{r+c(a, M)} \operatorname{det} M
$$

over all matrices $M \in G_{a}(A)$ such that $A_{p_{1}}, \ldots, A_{p_{m}}$ are the columns of $M$. The calculations

$$
\begin{aligned}
\mathcal{S} & \left(p_{1}, \ldots, p_{m}\right) \\
= & \sum_{j=1}^{m-1}(-1)^{r+\sum_{i=1}^{m} p_{i}+\left(p_{j}+n\right)} \operatorname{det}\left[A_{p_{1}}, \ldots, A_{p_{j-1}}, A_{p_{j+1}}, \ldots, A_{p_{m}}, A_{p_{j}}\right] \\
& +(-1)^{r+\sum_{i=1}^{m-1} p_{i}+\left(p_{m}+n\right)} \operatorname{det}\left[A_{p_{1}}, \ldots, A_{p_{m-1}}, A_{p_{m}}\right] \\
& = \begin{cases}(-1)^{r+\sum_{i=1}^{m} p_{i}+n} \operatorname{det}\left[A_{p_{1}}, \ldots, A_{p_{m}}\right], & \text { if } m \text { is odd and } p_{1}<\ldots<p_{m}, \\
0, & \text { otherwise }\end{cases}
\end{aligned}
$$

yield

$$
\begin{aligned}
\sum_{M \in G_{a}(A)}(-1)^{r+c(a, M)} \operatorname{det} M & =\sum_{p_{1}<\ldots<p_{m}} \mathcal{S}\left(p_{1}, \ldots, p_{m}\right) \\
& = \begin{cases}(-1)^{n} \operatorname{det} A, & \text { if } m \text { is odd }, \\
0, & \text { if } m \text { is even. }\end{cases}
\end{aligned}
$$

Thus, applying (5.1e), we obtain (5.2).

(f) Let $k=2$ and $a=(u, v)$, where $u \geq 2, v \geq 2$ are integers and $u+v=m$. In this case we prove (5.2) by induction. Assume that for every $\widetilde{m} \times n$ matrix $\widetilde{A}$ and for every $\widetilde{a}=(u-1, \widetilde{v})$, where $\widetilde{v} \geq 2$ is an integer and $u+\widetilde{v}=\widetilde{m}<m$, we have

$$
\sum_{M \in G_{\widetilde{a}}(\widetilde{A})}(-1)^{\widetilde{r}+c(\widetilde{a}, M)} \operatorname{det} M=S_{\widetilde{a}}^{\widetilde{m}} \operatorname{det} \widetilde{A},
$$

where $\widetilde{r}=1+2+\ldots+\widetilde{m}$.

Notice that

$$
\sum_{M \in G_{a}(A)}(-1)^{r+c(a, M)} \operatorname{det} M=\sum_{1 \leq p_{1}<\ldots<p_{m} \leq n}\left(\sum_{j=1}^{v+1} \mathcal{S}_{j}\left(p_{1}, \ldots, p_{m}\right)\right),
$$

where

$$
\mathcal{S}_{j}\left(p_{1}, \ldots, p_{m}\right)=\sum(-1)^{r+c(a, M)} \operatorname{det} M
$$

is the sum over all matrices $M \in G_{a}(A)$ such that $A_{p_{j}}$ is the first column of $M$ and the columns $A_{p_{i}}$, where $i \in\{1,2, \ldots, m\} \backslash\{j\}$, are the other columns 
of $M$. Moreover, for $1 \leq p_{1}<\ldots<p_{m} \leq n$, we have

$$
\begin{aligned}
& \mathcal{S}_{j}\left(p_{1}, \ldots, p_{m}\right)=\sum_{\substack{1 \leq r_{1}<\ldots<r_{u} \leq n \\
1 \leq r_{u+1}<\ldots<r_{m} \leq n \\
r_{1}=p_{j}}}(-1)^{r+\sum_{i=1}^{u} r_{i}+\sum_{i=u+1}^{m}\left(r_{i}+n\right)} \operatorname{det}\left[A_{r_{1}}, \ldots, A_{r_{m}}\right] \\
& \left\{r_{2}, \ldots, r_{m}\right\}=\left\{p_{1}, \ldots, p_{m}\right\} \backslash\left\{p_{j}\right\} \\
& =\sum_{\substack{1 \leq r_{1}<\ldots<r_{u} \leq n \\
1 \leq r_{u+1}<\ldots<r_{m} \leq n \\
r_{1}=p_{j} \\
\left\{r_{2}, \ldots, r_{m}\right\}=\left\{p_{1}, \ldots, p_{m}\right\} \backslash\left\{p_{j}\right\}}}(-1)^{r+\sum_{i=1}^{m} p_{i}+n v+\ell\left(\left[p_{j}, r_{2}, \ldots, r_{m}\right],\left[p_{1}, \ldots, p_{m}\right]\right)} \operatorname{det}\left[A_{p_{1}}, \ldots, A_{p_{m}}\right], \\
&
\end{aligned}
$$

where $\ell\left(\left[p_{j}, r_{2}, \ldots, r_{m}\right],\left[p_{1}, \ldots, p_{m}\right]\right)$ is the number of interchanges of two adjacent columns that should be performed to obtain $\left[A_{p_{1}}, \ldots, A_{p_{m}}\right]$ from $\left[A_{p_{j}}, A_{r_{2}}, \ldots, A_{r_{m}}\right]$. Since

$$
\begin{aligned}
& \ell\left(\left[p_{j}, r_{2}, \ldots, r_{m}\right],\left[p_{1}, \ldots, p_{m}\right]\right) \\
&=\ell\left(\left[p_{j}, r_{2}, \ldots, r_{m}\right],\left[p_{1}, \ldots, p_{j}, r_{2}, \ldots, r_{u}, r_{u+j}, \ldots, r_{m}\right]\right) \\
&+\ell\left(\left[p_{1}, \ldots, p_{j}, r_{2}, \ldots, r_{u}, r_{u+j}, \ldots, r_{m}\right],\left[p_{1}, \ldots, p_{m}\right]\right) \\
&=(j-1) u+\ell\left(\left[r_{2}, \ldots, r_{u}, r_{u+j}, \ldots, r_{m}\right],\left[p_{j+1}, \ldots, p_{m}\right]\right),
\end{aligned}
$$

we have

$$
\mathcal{S}_{j}\left(p_{1}, \ldots, p_{m}\right)=(-1)^{r+\sum_{i=1}^{m} p_{i}} \operatorname{det}\left[A_{p_{1}}, \ldots, A_{p_{m}}\right](-1)^{n v+(j-1) u} Z_{j},
$$

where

$$
Z_{j}=\sum_{\substack{1 \leq r_{2}<\ldots<r_{u} \leq n \\ 1 \leq r_{u+j}<\ldots<r_{m} \leq n \\\left\{r_{2}, \ldots, r_{u}, r_{u+j}, \ldots, r_{m}\right\}=\left\{p_{j+1}, \ldots, p_{m}\right\}}}(-1)^{\ell\left(\left[r_{2}, \ldots, r_{u}, r_{u+j}, \ldots, r_{m}\right],\left[p_{j+1}, \ldots, p_{m}\right]\right)} .
$$

The number $Z_{j}$ depends only on the number of elements in $\left\{p_{j+1}, \ldots, p_{m}\right\}$ and does not depend on the numbers $p_{1}, \ldots, p_{m}$, therefore

$$
\sum_{M \in G_{a}(A)}(-1)^{r+c(a, M)} \operatorname{det} M=\operatorname{det} A \cdot \sum_{j=1}^{v+1}(-1)^{(n+u)(j-1)}(-1)^{n(v-(j-1))} Z_{j} .
$$

For each $j \in\{1,2, \ldots, v+1\}$ define $\widetilde{r}_{j}=1+2+\ldots+(m-j)$ and consider a $(m-j) \times n$ matrix $\widetilde{\mathcal{A}}^{(j)}=\left[\widetilde{\mathcal{A}}_{1}^{(j)}, \ldots, \widetilde{\mathcal{A}}_{n}^{(j)}\right]$ with columns $\widetilde{\mathcal{A}}_{1}^{(j)}, \ldots, \widetilde{\mathcal{A}}_{n}^{(j)}$. 
We have

$$
\begin{aligned}
& \operatorname{det} \widetilde{\mathcal{A}}^{(j)} \cdot(-1)^{n(v-(j-1))} Z_{j} \\
& =\sum_{1 \leq p_{j+1}<\ldots<p_{m} \leq n}(-1)^{\widetilde{r}_{j}+\sum_{i=j+1}^{m} p_{i}} \operatorname{det}\left[\widetilde{\mathcal{A}}_{p_{j+1}}^{(j)}, \ldots, \widetilde{\mathcal{A}}_{p_{m}}^{(j)}\right](-1)^{n(v-(j-1))} Z_{j} \\
& =\sum_{1 \leq p_{j+1}<\ldots<p_{m} \leq n} \sum_{\substack{1 \leq r_{2}<\ldots<r_{u} \leq n \\
1 \leq r_{u+j}<\ldots<r_{m} \leq n}}(-1)^{\widetilde{r}_{j}+\sum_{i=2}^{u} r_{i}+\sum_{i=u+j}^{m}\left(r_{i}+n\right)} \\
& \left\{r_{2}, \ldots, r_{u}, r_{u+j}, \ldots, r_{m}\right\}=\left\{p_{j+1}, \ldots, p_{m}\right\} \\
& \times \operatorname{det}\left[\widetilde{\mathcal{A}}_{r_{2}}^{(j)}, \ldots, \widetilde{\mathcal{A}}_{r_{u}}^{(j)}, \widetilde{\mathcal{A}}_{r_{u+j}}^{(j)}, \ldots, \widetilde{\mathcal{A}}_{r_{m}}^{(j)}\right] \\
& =\sum(-1)^{\widetilde{r}_{j}+c((u-1, v-j+1), M)} \operatorname{det} M \\
& M \in G_{(u-1, v-j+1)}\left(\widetilde{\mathcal{A}}^{(j)}\right) \\
& =S_{(u-1, v-j+1)}^{\widetilde{n}} \cdot \operatorname{det} \widetilde{\mathcal{A}}^{(j)} .
\end{aligned}
$$

Finally, applying (5.1f), we obtain

$$
\begin{aligned}
\sum_{M \in G_{a}(A)}(-1)^{r+c(a, M)} \operatorname{det} M & =\operatorname{det} A \cdot \sum_{j=1}^{v+1}(-1)^{(n+u)(j-1)} S_{(u-1, v-j+1)}^{\widetilde{n}} \\
& =\operatorname{det} A \cdot S_{a}^{\widetilde{n}}
\end{aligned}
$$

(g) For an arbitrary integer $k \geq 3$ and $a=\left(a_{1}, \ldots, a_{p}, 0, \ldots, 0\right)$, where $p<k$ and $a_{1} \neq 0, a_{p} \neq 0$, we have $G_{a}(A)=G_{\left(a_{1}, \ldots, a_{p}\right)}(A)$ and $c(a, M)=$ $c\left(\left(a_{1}, \ldots, a_{p}\right), M\right)$ for every $M \in G_{a}(A)$. Therefore, applying (5.1g), we obtain

$$
\begin{aligned}
\sum_{M \in G_{a}(A)}(-1)^{r+c(a, M)} \operatorname{det} M & =\sum_{M \in G_{\left(a_{1}, \ldots, a_{p}\right)}(A)}(-1)^{r+c\left(\left(a_{1}, \ldots, a_{p}\right), M\right)} \operatorname{det} M \\
& =S_{\left(a_{1}, \ldots, a_{p}\right)}^{\widetilde{n}} \operatorname{det} A=S_{a}^{\widetilde{n}} \operatorname{det} A .
\end{aligned}
$$

(h) Let $k \geq 3$ be an integer and $a=\left(a_{1}, \ldots, a_{k}\right)$, where $p<k, a_{1}=\ldots=$ $a_{p}=0$ and $a_{p+1} \neq 0$. For every matrix $M=\left[M_{1}, \ldots, M_{m}\right] \in G_{a}(A)$ such that $M_{i}=B_{j_{i}}$ for each $i=1,2, \ldots, m$, we have $M \in G_{\left(a_{p+1}, \ldots, a_{k}\right)}(A)$ and

$$
c(a, M)=\sum_{i=1}^{m} j_{i}=\sum_{i=1}^{m}\left(j_{i}-n p\right)+m n p=c\left(\left(a_{p+1}, \ldots, a_{k}\right), M\right)+m n p .
$$


Since there is a one-to-one correspondence between matrices in $G_{a}(A)$ and $G_{\left(a_{p+1}, \ldots, a_{k}\right)}(A)$, we have

$$
\begin{aligned}
\sum_{M \in G_{a}(A)} & (-1)^{r+c(a, M)} \operatorname{det} M \\
& =(-1)^{m n p} \sum_{M \in G_{\left(a_{p+1}, \ldots, a_{k}\right)}(A)}(-1)^{r+c\left(\left(a_{p+1}, \ldots, a_{k}\right), M\right)} \operatorname{det} M \\
& =(-1)^{m n p} \cdot S_{\left(a_{p+1}, \ldots, a_{k}\right)}^{\tilde{n}} \operatorname{det} A .
\end{aligned}
$$

Now, applying (5.1h), we get (5.2).

(i) Let $k \geq 3$ be an integer and $a=\left(a_{1}, \ldots, a_{k}\right)$, where $a_{1} \neq 0$ and $a_{p} \neq 0$. Notice that

$$
\sum_{M \in G_{a}(A)}(-1)^{r+c(a, M)} \operatorname{det} M=\sum_{1 \leq p_{1}<\ldots<p_{m} \leq n} \mathcal{S}\left(p_{1}, \ldots, p_{m}\right),
$$

where

$$
\mathcal{S}\left(p_{1}, \ldots, p_{m}\right)=\sum(-1)^{r+c(a, M)} \operatorname{det} M
$$

is the sum over all matrices $M \in G_{a}(A)$ such that $A_{p_{1}}, A_{p_{2}}, \ldots, A_{p_{m}}$ are the columns of $M$. Let $b=\left(b_{1}, \ldots, b_{k-1}\right)$, where $b_{i}=a_{i}$ for $i=1,2, \ldots, k-2$ and $b_{k-1}=a_{k-1}+a_{k}$, For $1 \leq p_{1}<\ldots<p_{m} \leq n$, we have

$$
\begin{aligned}
& \mathcal{S}\left(p_{1}, \ldots, p_{m}\right)=\sum_{1 \leq r_{1}<\ldots<r_{a_{1}} \leq n}(-1)^{r+\sum_{i=1}^{k} r_{i}+\sum_{i=2}^{k} a_{i}(i-1) n} \operatorname{det}\left[A_{r_{1}}, \ldots, A_{r_{m}}\right] \\
& 1 \leq r_{a_{1}+1}<\ldots<r_{a_{1}+a_{2}} \leq n \\
& 1 \leq r_{a_{1}+\ldots+a_{k-1}+1}<\ldots<r_{m} \leq n \\
& \left\{r_{1}, \ldots, r_{m}\right\}=\left\{p_{1}, \ldots, p_{m}\right\} \\
& =\sum(-1)^{r+\sum_{i=1}^{m} p_{i}+\sum_{i=2}^{k} b_{i}(i-1) n+a_{k} n} \\
& \times(-1)^{\ell\left(\left[r_{b_{1}+\ldots+b_{k-2}+1}, \ldots, r_{m}\right],\left[\widetilde{p}_{b_{1}+\ldots+b_{k-2}+1}, \ldots, \widetilde{p}_{m}\right]\right)} \\
& \times \operatorname{det}\left[A_{r_{1}}, \ldots, A_{r_{b_{1}+\ldots+b_{k-2}}}, A_{\widetilde{p}_{b_{1}+\ldots+b_{k-2}+1}}, \ldots, A_{\widetilde{p}_{m}}\right],
\end{aligned}
$$

where the last sum is taken over the indices satisfying the following relations:

$$
\begin{aligned}
& 1 \leq r_{1}<\ldots<r_{a_{1}} \leq n, \quad 1 \leq r_{a_{1}+1}<\ldots<r_{a_{1}+a_{2}} \leq n, \ldots, \\
& 1 \leq r_{a_{1}+\ldots+a_{k-1}+1}<\ldots<r_{m} \leq n, \\
& \left\{r_{1}, \ldots, r_{m}\right\}=\left\{p_{1}, \ldots, p_{m}\right\} \\
& 1 \leq \widetilde{p}_{b_{1}+\ldots+b_{k-2}+1}<\ldots<\widetilde{p}_{m} \leq n, \\
& \left\{\widetilde{p}_{b_{1}+\ldots+b_{k-2}+1}, \ldots, \widetilde{p}_{m}\right\}=\left\{r_{b_{1}+\ldots+b_{k-2}+1}, \ldots, r_{m}\right\} .
\end{aligned}
$$


Since the sum

$$
\begin{aligned}
& Z=\sum_{1 \leq \widetilde{p}_{b_{1}+\ldots+b_{k-2}+1}<\ldots<\widetilde{p}_{m} \leq n}(-1)^{\ell\left(\left[r_{b_{1}+\ldots+b_{k-2}+1}, \ldots, r_{m}\right],\left[\widetilde{p}_{b_{1}+\ldots+b_{k-2}+1}, \ldots, \widetilde{p}_{m}\right]\right)} \\
& \left\{r_{b_{1}+\ldots+b_{k-2}+1}, \ldots, r_{m}\right\}=\left\{\widetilde{p}_{b_{1}+\ldots+b_{k-2}+1}, \ldots, \widetilde{p}_{m}\right\} \\
& 1 \leq r_{b_{1}+\ldots+b_{k-2}}<\ldots<r_{b_{1}+\ldots+b_{k-1}} \leq n \\
& 1 \leq r_{b_{1}+\ldots+b_{k-1}+1}<\ldots<r_{m} \leq n
\end{aligned}
$$

does not depend on the numbers $p_{1}, \ldots, p_{m}, \widetilde{p}_{1}, \ldots, \widetilde{p}_{m}$ (it depends only on the number of elements in $\left.\left\{\widetilde{p}_{b_{1}+\ldots+b_{k-2}+1}, \ldots, \widetilde{p}_{m}\right\}\right)$, we have

$$
\begin{aligned}
\mathcal{S}\left(p_{1}, \ldots, p_{m}\right)=\sum & (-1)^{r+\sum_{i=1}^{k} p_{i}+\sum_{i=2}^{n-1} b_{i}(i-1) n} \\
& \times \operatorname{det}\left[A_{r_{1}}, \ldots, A_{r_{b_{1}+\ldots+b_{k-2}}}, A_{\widetilde{p}_{b_{1}+\ldots+b_{k-2}+1}}, \ldots, A_{\widetilde{p}_{m}}\right] \\
& \times(-1)^{a_{k} n} Z,
\end{aligned}
$$

where the sum is taken over the indices satisfying the following relations:

$$
\begin{aligned}
& 1 \leq r_{1}<\ldots<r_{b_{1}} \leq n, \quad 1 \leq r_{b_{1}+1}<\ldots<r_{b_{1}+b_{2}} \leq n, \ldots, \\
& 1 \leq r_{b_{1} \ldots+b_{k-3}+1}<\ldots<r_{b_{1} \ldots+b_{k-2}} \leq n, \\
& \left\{r_{1}, \ldots, r_{m}\right\}=\left\{p_{1}, \ldots, p_{m}\right\}, \\
& 1 \leq \widetilde{p}_{b_{1}+\ldots+b_{k-2}+1}<\ldots<\widetilde{p}_{m} \leq n, \\
& \left\{\widetilde{p}_{b_{1}+\ldots+b_{k-2}+1}, \ldots, \widetilde{p}_{m}\right\}=\left\{r_{b_{1}+\ldots+b_{k-2}+1}, \ldots, r_{m}\right\} .
\end{aligned}
$$

Assuming that (5.2) is true for any sequence of $k-1$ nonnegative integers which sum is equal to $m$, we get

$$
\begin{aligned}
\sum_{M \in G_{a}(A)}(-1)^{r+c(a, M)} \operatorname{det} M & =\sum_{M \in G_{b}(A)}(-1)^{r+c(b, M)} \operatorname{det} M \cdot(-1)^{a_{k} n} Z \\
& =\operatorname{det} A \cdot S_{b}^{\widetilde{n}} \cdot(-1)^{a_{k} n} Z .
\end{aligned}
$$

For every matrix $M \in G_{\left(a_{k}-1, a_{k}\right)}$ we have

$$
c\left(\left(a_{k-1}, a_{k}\right), M\right)=c\left(\left(a_{k-1}+a_{k}\right), M\right)+a_{k} n=c\left(\left(b_{k-1}\right), M\right)+a_{k} n .
$$

Therefore, for every $b_{k-1} \times n$ matrix $\mathcal{A}$, calculations similar to those in (5.3) yield

and hence

$$
\operatorname{det} \mathcal{A} \cdot(-1)^{a_{k} n} Z=\operatorname{det} \mathcal{A} \cdot S_{\left(a_{k-1}, a_{k}\right)}^{\widetilde{n}}
$$

$$
(-1)^{a_{k} n} Z=S_{\left(a_{k-1}, a_{k}\right)}^{\widetilde{n}} .
$$

Finally, applying (5.1i), we obtain

$$
\begin{aligned}
\sum_{M \in G_{a}(A)}(-1)^{r+c(a, M)} \operatorname{det} M & =\operatorname{det} A \cdot S_{b}^{\widetilde{n}} \cdot(-1)^{a_{k} n} Z \\
& =\operatorname{det} A \cdot S_{b}^{\widetilde{n}} \cdot S_{\left(a_{k-1}, a_{k}\right)}^{\widetilde{n}}=\operatorname{det} A \cdot S_{a}^{\widetilde{n}} .
\end{aligned}
$$


The next two lemmas give explicit formulas for $S_{\left(a_{1}, \ldots, a_{k}\right)}^{\widetilde{n}}$.

Lemma 5.4. Let $n$ and $m$ be positive integers, $\widetilde{n}=\frac{1-(-1)^{n}}{2}$ and $m=p+q$, where $p, q$ are nonnegative integers.

(a) If $p \not \equiv q(\bmod 2)$, then

$$
S_{(p, q)}^{\widetilde{n}}=\left(\begin{array}{c}
\frac{p+q-1}{2} \\
\left\lfloor\frac{p}{2}\right\rfloor
\end{array}\right)(-1)^{(p+1) n}=\left(\begin{array}{c}
\frac{p+q-1}{2} \\
\left\lfloor\frac{q}{2}\right\rfloor
\end{array}\right)(-1)^{q n}=\left(\begin{array}{c}
\frac{p+q-1}{2} \\
\left\lfloor\frac{p}{2}\right\rfloor,\left\lfloor\frac{q}{2}\right\rfloor
\end{array}\right)(-1)^{q n} .
$$

(b) If both $p$ and $q$ are even, then

$$
S_{(p, q)}^{\widetilde{n}}=\left(\begin{array}{c}
\frac{p+q}{2} \\
\frac{p}{2}
\end{array}\right)=\left(\begin{array}{c}
\frac{p+q}{2} \\
\frac{q}{2}
\end{array}\right)=\left(\begin{array}{c}
\frac{p+q}{2} \\
\frac{p}{2}, \frac{q}{2}
\end{array}\right) .
$$

(c) If both $p$ and $q$ are odd, then

$$
S_{(p, q)}^{\widetilde{n}}=0 .
$$

Proof. The proof will be done by induction. If $p<2$ or $q<2$, then (5.4), (5.5) and (5.6) follow from (5.1b), (5.1c), (5.1d), (5.1e) and are easy to verify. For $p \geq 2$ and $q \geq 2$, in each of the following cases we apply (5.1f) first.

$\left(\mathrm{a}_{1}\right)$ If $p$ is odd and $q$ is even, we have

$$
\begin{aligned}
S_{(p, q)}^{\widetilde{n}} & =\left(\begin{array}{c}
\frac{p+q-1}{2} \\
\frac{p-1}{2}
\end{array}\right)+\sum_{j=1}^{q / 2}\left[(-1)^{(2 j-1)(p+n)} S_{(p-1, q-2 j+1)}^{\widetilde{n}}+(-1)^{2 j(p+n)} S_{(p-1, q-2 j)}^{\widetilde{n}}\right] \\
& =\left(\begin{array}{c}
\frac{p+q-1}{2} \\
\frac{p-1}{2}
\end{array}\right)+\sum_{j=1}^{q / 2}\left[-\left(\begin{array}{c}
\frac{p+q-1}{2}-j \\
\left\lfloor\frac{p-1}{2}\right\rfloor
\end{array}\right)+\left(\begin{array}{c}
\frac{p+q-1}{2}-j \\
\frac{p-1}{2}
\end{array}\right)\right] \\
& =\left(\begin{array}{c}
\frac{p+q-1}{2} \\
\left\lfloor\frac{p-1}{2}\right\rfloor
\end{array}\right)(-1)^{(p+1) n} .
\end{aligned}
$$

$\left(\mathrm{a}_{2}\right)$ If $p$ is even and $q$ is odd, then

$$
\begin{aligned}
S_{(p, q)}^{\widetilde{n}} & =\sum_{i=0}^{q}(-1)^{i(p+n)} S_{(p-1, q-i)}^{\widetilde{n}}=\sum_{j=0}^{(q-1) / 2}(-1)^{(2 j+1)(p+n)} S_{(p-1, q-2 j-1)}^{\widetilde{n}} \\
& =\sum_{j=0}^{(q-1) / 2}(-1)^{n}\left(\begin{array}{c}
\frac{p+q-3}{2}-j \\
\left\lfloor\frac{p-1}{2}\right\rfloor
\end{array}\right)=\left(\begin{array}{c}
\frac{p+q-3}{2}+1 \\
\left\lfloor\frac{p-1}{2}\right\rfloor+1
\end{array}\right)(-1)^{n} \\
& =\left(\begin{array}{c}
\frac{p+q-1}{2} \\
\left\lfloor\frac{p}{2}\right\rfloor
\end{array}\right)(-1)^{(p+1) n} .
\end{aligned}
$$


(b) If both $p$ and $q$ are even, we obtain

$$
\begin{aligned}
S_{(p, q)}^{\widetilde{n}} & =\sum_{j=0}^{q / 2}(-1)^{2 j(p+n)} S_{(p-1, q-2 j)}^{\widetilde{n}}=\sum_{j=0}^{q / 2}(-1)^{p n}\left(\begin{array}{c}
\frac{p+q-2}{2}-j \\
\left\lfloor\frac{p-1}{2}\right\rfloor
\end{array}\right) \\
& =\left(\begin{array}{c}
\frac{p+q-2}{2}+1 \\
\left\lfloor\frac{p-1}{2}\right\rfloor+1
\end{array}\right)=\left(\begin{array}{c}
\frac{p+q}{2} \\
\frac{p}{2}
\end{array}\right) .
\end{aligned}
$$

(c) If both $p$ and $q$ are odd, we have

$$
\begin{aligned}
S_{(p, q)}^{\widetilde{n}} & =\sum_{j=0}^{(q-1) / 2}\left[(-1)^{2 j(p+n)} S_{(p-1, q-2 j)}^{\widetilde{n}}+(-1)^{(2 j+1)(p+n)} S_{(p-1, q-2 j-1)}^{\widetilde{n}}\right] \\
& =\sum_{j=0}^{(q-1) / 2}\left[(-1)^{n}\left(\begin{array}{c}
\frac{p+q-2}{2}-j \\
\left.\frac{p-1}{2}\right\rfloor
\end{array}\right)+(-1)^{n+1}\left(\begin{array}{c}
\frac{p+q-2}{2}-j \\
\frac{p-1}{2}
\end{array}\right)\right]=0 .
\end{aligned}
$$

Remark 5.5. In $[4, \S 33$ and $\S 34]$, Cullis proved the following formula

$$
Q_{k-m}^{n-m} \operatorname{det} A=\sum_{1 \leq j_{1} \leq \ldots \leq j_{k} \leq n}(-1)^{\sum_{i=1}^{k}\left(j_{i}-i\right)} \operatorname{det}\left[A_{j_{1}}, \ldots, A_{j_{k}}\right],
$$

where $1 \leq m \leq k \leq n, A=\left[A_{1}, \ldots, A_{n}\right]$ is an $m \times n$ matrix and the numbers $Q_{k-m}^{n-m}$ are defined by

$$
Q_{2 m}^{2 n}=Q_{2 m+1}^{2 n+1}=Q_{2 m}^{2 n+1}=\left(\begin{array}{c}
n \\
m
\end{array}\right) \quad \text { and } \quad Q_{2 m+1}^{2 n+2}=0,
$$

where $m, n$ are nonnegative integers and $m \leq n$. From Lemma 5.4 it follows that

$$
Q_{k-m}^{n-m}=S_{(k-m, n-k)}^{0}=S_{(n-k, k-m)}^{0} .
$$

Lemma 5.6. Let $k, m$ and $n$ be positive integers, $\widetilde{n}=\frac{1-(-1)^{n}}{2}$ and $m=$ $\sum_{i=1}^{k} a_{i}$ be a sum of nonnegative integers.

(A) If all the numbers $a_{1}, \ldots, a_{k}$ are even, then

$$
S_{\left(a_{1}, \ldots, a_{k}\right)}^{\widetilde{n}}=\left(\begin{array}{c}
\frac{a_{1}+\ldots+a_{k}}{2} \\
\frac{a_{1}}{2}, \ldots, \frac{a_{k}}{2}
\end{array}\right) .
$$

(B) If there is exactly one odd number $a_{p}$ among $a_{1}, \ldots, a_{k}$, then

$$
S_{\left(a_{1}, \ldots, a_{k}\right)}^{\widetilde{n}}=\left(\begin{array}{c}
\frac{a_{1}+\ldots+a_{k}-1}{2}\left\lfloor\frac{a_{1}}{2}\right\rfloor, \ldots,\left\lfloor\frac{a_{k}}{2}\right\rfloor
\end{array}\right)(-1)^{(p+1) n} .
$$

(C) If there are at least two odd numbers among $a_{1}, \ldots, a_{k}$, then

$$
S_{\left(a_{1}, \ldots, a_{k}\right)}^{\widetilde{n}}=0
$$


Proof. The proof will be done by induction on $k$.

For $k=1$, we consider only (5.7) and (5.8). They easily follow from (5.1a). For $k=2$, formulas (5.7), (5.8) and (5.9) follow from Lemma 5.4. (Note that $p$ in (5.4) and $p$ in (5.8) are generally not equal, but they have the same parity if $k=2$.) For $k \geq 3$, consider the following cases.

(A) If all the numbers $a_{1}, \ldots, a_{k}$ are even, we apply (5.1i) and obtain

$$
\begin{aligned}
S_{\left(a_{1}, \ldots, a_{k}\right)}^{\widetilde{n}} & =S_{\left(a_{1}, \ldots, a_{k-2}, a_{k-1}+a_{k}\right)}^{\widetilde{n}} S_{\left(a_{k-1}, a_{k}\right)}^{\widetilde{n}} \\
& =\left(\begin{array}{c}
\frac{a_{1}+\ldots+a_{k-2}+a_{k-1}+a_{k}}{2} \\
\frac{a_{1}}{2}, \ldots, \frac{a_{k-2}}{2}, \frac{a_{k-1}+a_{k}}{2}
\end{array}\right)\left(\begin{array}{l}
\frac{a_{k-1}+a_{k}}{2} \\
\frac{a_{k-1}}{2}, \frac{a_{k}}{2}
\end{array}\right)=\left(\begin{array}{c}
\frac{a_{1}+\ldots+a_{k}}{2} \\
\frac{a_{1}}{2}, \ldots, \frac{a_{k}}{2}
\end{array}\right) .
\end{aligned}
$$

(B) If $a_{p}$ is odd for some $p \in\{1,2, \ldots, k\}$ and $a_{i}$ is even for each $i \in$ $\{1,2, \ldots, k\} \backslash\{p\}$, consider two subcases.

$\left(\mathrm{B}_{1}\right)$ If $p<k-1$, we apply (5.1i) and obtain

$$
\begin{aligned}
S_{\left(a_{1}, \ldots, a_{k}\right)}^{\widetilde{n}} & =\left(\begin{array}{c}
\frac{a_{1}+\ldots+a_{k}-1}{2} \\
\left\lfloor\frac{a_{1}}{2}\right\rfloor, \ldots,\left\lfloor\frac{a_{k-1}+a_{k}}{2}\right\rfloor
\end{array}\right)(-1)^{(p+1) n(}\left(\begin{array}{c}
\frac{a_{k-1}+a_{k}}{2} \\
\frac{a_{k-1}}{2}, \frac{a_{k}}{2}
\end{array}\right) \\
& =\left(\begin{array}{c}
\frac{a_{1}+\ldots+a_{k}-1}{2} \\
\left\lfloor\frac{a_{1}}{2}\right\rfloor, \ldots,\left\lfloor\frac{a_{k}}{2}\right\rfloor
\end{array}\right)(-1)^{(p+1) n} .
\end{aligned}
$$

$\left(\mathrm{B}_{2}\right)$ Similarly, if $p-k=0$ or $p-k=-1$, we have

$$
\begin{aligned}
S_{\left(a_{1}, \ldots, a_{k}\right)}^{\widetilde{\widetilde{n}}} & =\left(\begin{array}{c}
\frac{a_{1}+\ldots+a_{k}-1}{2} \\
\left\lfloor\frac{a_{1}}{2}\right\rfloor, \ldots,\left\lfloor\frac{a_{k-1}+a_{k}}{2}\right\rfloor
\end{array}\right)(-1)^{k n}\left(\begin{array}{c}
\frac{a_{k-1}+a_{k}-1}{2} \\
\left\lfloor\frac{a_{k-1}}{2}\right\rfloor,\left\lfloor\frac{a_{k}}{2}\right\rfloor
\end{array}\right)(-1)^{(p-k+3) n} \\
& =\left(\begin{array}{c}
\frac{a_{1}+\ldots+a_{k}-1}{2} \\
\left\lfloor\frac{a_{1}}{2}\right\rfloor, \ldots,\left\lfloor\frac{a_{k}}{2}\right\rfloor
\end{array}\right)(-1)^{(p+1) n} .
\end{aligned}
$$

(C) If there are at least two odd numbers among $a_{1}, \ldots, a_{k}$, let $a_{p}$ and $a_{q}$, where $p<q, p, q \in\{1,2, \ldots, k\}$, be the two largest odd numbers in $\left\{a_{1}, \ldots, a_{k}\right\}$. Consider two subcases.

$\left(\mathrm{C}_{1}\right)$ If $p>1$, we apply (5.1i) $k-p$ times and obtain

$$
\begin{aligned}
S_{\left(a_{1}, \ldots, a_{k}\right)}^{\widetilde{n}}= & S_{\left(a_{1}, \ldots, a_{p-1}, a_{p}+a_{p+1}+\ldots+a_{k}\right)}^{\widetilde{n}} \\
& \times S_{\left(a_{p}, a_{p+1}+\ldots+a_{k}\right)}^{\widetilde{n}} \ldots S_{\left(a_{k-2}, a_{k-1}+a_{k}\right)}^{\widetilde{n}} S_{\left(a_{k-1}, a_{k}\right)}^{\widetilde{n}} .
\end{aligned}
$$

The numbers $a_{p}$ and $a_{p+1}+\ldots+a_{n}$ are odd, so $S_{\left(a_{p}, a_{p+1}+\ldots+a_{n}\right)}^{\widetilde{n}}=0$ and consequently $S_{\left(a_{1}, \ldots, a_{n}\right)}^{\widetilde{n}}=0$.

$\left(\mathrm{C}_{2}\right)$ If $p=1$, then $S_{\left(a_{1}, a_{2}+\ldots+a_{k}\right)}^{\widetilde{n}}=0$ and after applying $(5.1 \mathrm{i}) k-2$ times, we have

$$
S_{\left(a_{1}, \ldots, a_{k}\right)}^{\widetilde{n}}=S_{\left(a_{1}, a_{2}+\ldots+a_{k}\right)}^{\widetilde{n}} S_{\left(a_{2}, a_{3}+\ldots+a_{k}\right)}^{\widetilde{n}} \ldots S_{\left(a_{k-2}, a_{k-1}+a_{k}\right)}^{\widetilde{n}} S_{\left(a_{k-1}, a_{k}\right)}^{\widetilde{n}}=0 .
$$


Lemma 5.7. Let $k, m, n$ be positive integers and $\widetilde{n}=\frac{1-(-1)^{n}}{2}$. Then (5.10)

$$
\sum_{\substack{\left(a_{1}, \ldots, a_{k}\right) \\ a_{1}+\ldots+a_{k}=m}} S_{\left(a_{1}, \ldots, a_{k}\right)}^{\widetilde{n}}= \begin{cases}k^{m / 2}, & \text { if } m \text { is even } \\ k^{(m+1) / 2}, & \text { if } m \text { is odd and } n \text { is even, } \\ 0, & \text { if } m, n \text { are odd and } k \text { is even }, \\ k^{(m-1) / 2}, & \text { if } m, n, k \text { are odd. }\end{cases}
$$

Proof. Denote the left-hand side of (5.10) by $L$ and consider two cases.

(i) If $m$ is even, we apply Lemma $5.6(\mathrm{C})$ and Lemma $5.6(\mathrm{~A})$, and obtain

$$
\begin{aligned}
& L=\sum_{\substack{\left(a_{1}, \ldots, a_{k}\right) \\
a_{1}+\ldots+a_{k}=m}} S_{\left(a_{1}, \ldots, a_{k}\right)}^{\widetilde{n}}=\sum_{\begin{array}{c}
\left(a_{1}, \ldots, a_{k}\right) \\
a_{1}+\ldots+a_{k}=m
\end{array}}\left(\begin{array}{c}
\frac{a_{1}+\ldots+a_{k}}{2} \\
\frac{a_{1}}{2}, \ldots, \frac{a_{k}}{2}
\end{array}\right)=k^{\frac{m}{2}}, \\
& a_{i} \geq 0, a_{i} \equiv 0(\bmod 2), i=1,2, \ldots, k \quad a_{i} \geq 0, a_{i} \equiv 0(\bmod 2), i=1,2, \ldots, k
\end{aligned}
$$

(ii) If $m$ is odd, by $\mathcal{A}$ we denote the set of all sequences of nonnegative integers $\left(a_{1}, \ldots, a_{k}\right)$ containing exactly one odd number and satisfying $\sum_{i=1}^{k} a_{i}=m$. By Lemma $5.6(\mathrm{~B})$, we have

$$
\begin{aligned}
L & =\sum_{p=1}^{k} \sum_{\substack{\left(a_{1}, \ldots, a_{k}\right) \in \mathcal{A} \\
a_{p} \equiv 1(\bmod 2)}}\left(\begin{array}{c}
\frac{a_{1}+\ldots+a_{k}-1}{2} \\
\left\lfloor\frac{a_{1}}{2}\right\rfloor, \ldots,\left\lfloor\frac{a_{k}}{2}\right\rfloor
\end{array}\right)(-1)^{(p+1) n} \\
= & \sum_{p=1}^{k} \sum_{\substack{\left(a_{1}, \ldots, a_{k}\right) \in \mathcal{A} \\
a_{p} \equiv 1(\bmod 2)}}\left(\begin{array}{c}
\frac{a_{1}+\ldots+a_{p-1}+\left(a_{p}-1\right)+a_{p+1}+\ldots+a_{k}}{2} \\
\left.\frac{a_{1}}{2}, \ldots, \frac{a_{p-1}}{2}, \frac{\left(a_{p}-1\right)}{2}, \frac{a_{p+1}}{2}, \ldots, \frac{a_{k}}{2}\right)(-1)^{(p+1) n}
\end{array}\right. \\
& = \begin{cases}k \cdot k^{(m-1) / 2}, & \text { if } n \text { is even, } \\
\sum_{p=1}^{k}(-1)^{p+1} \cdot k^{(m-1) / 2}, & \text { if } n \text { is odd, }\end{cases} \\
& = \begin{cases}k^{(m+1) / 2}, & \text { if } n \text { is even, } \\
0, & \text { if } n \text { is odd and } k \text { is even, } \\
k^{(m-1) / 2}, & \text { if } n \text { is odd and } k \text { is odd. }\end{cases}
\end{aligned}
$$

Example 5.8. If $A=\left[A_{1}, A_{2}, A_{3}\right]$ is a $3 \times 3$ matrix and

$$
B=[A, A, A]=\left[A_{1}, A_{2}, A_{3}, A_{1}, A_{2}, A_{3}, A_{1}, A_{2}, A_{3}\right]
$$

is a $3 \times 9$ matrix, then

$$
\operatorname{det} B=3 \operatorname{det} A \text {. }
$$




\section{REFERENCES}

[1] Amiri, A., Fathy, M., Bayat, M., Generalization of some determinantal identities for non-square matrices based on Radic's definition, TWMS J. Pure Appl. Math. 1 (2) (2010), 163-175.

[2] Arunkumar, M., Murthy, S., Ganapathy, G., Determinant for non-square matrices, Int. J. Math. Sci. Eng. Appl. 5 (5) (2011), 389-401.

[3] Buraczewski, A., Generalization of formulae of Fredholm type and determinant theory for rectangular matrices, Panamer. Math. J. 1 (2) (1991), 49-66.

[4] Cullis, C. E., Matrices and Determinoids, Vol. 1, Cambridge University Press, Cambridge, 1913.

[5] Joshi, V. N., A determinant for rectangular matrices, Bull. Austral. Math. Soc. 21 (1) (1980), 137-146.

[6] Makarewicz, A., Pikuta, P., Szałkowski, D., Properties of the determinant of a rectangular matrix, Ann. Univ. Mariae Curie-Skłodowska Sect. A 68 (1) (2014), 31-41.

[7] Makarewicz, A., Mozgawa, W., Pikuta, P., Volumes of polyhedra in terms of determinants of rectangular matrices, Bull. Soc. Sci. Lett. Łódź Sér. Rech. Déform. 66 (2) (2016), 105-117.

[8] Nakagami, Y., Yanai, H., On Cullis' determinant for rectangular matrices, Linear Algebra Appl. 422 (2-3) (2007), 422-441.

[9] Pyle, H. R., Non-square determinants and multilinear vectors, Math. Mag. 35 (2) (1962), 65-69.

[10] Radić, M., A generalization of the determinant of a square matrix and some of its applications in geometry, Matematika (Zagreb) 20 (2) (1991), 19-36 (SerboCroatian).

[11] Radić, M., A definition of determinant of rectangular matrix, Glas. Mat. Ser. III 1(21) (1966), 17-22.

[12] Radić, M., About a determinant of rectangular $2 \times n$ matrix and its geometric interpretation, Beiträge Algebra Geom. 46 (2) (2005), 321-349.

[13] Radić, M., Areas of certain polygons in connection with determinants of rectangular matrices, Beiträge Algebra Geom. 49 (1) (2008), 71-96.

[14] Radić, M., Certain equalities and inequalities concerning polygons in $\mathbb{R}^{2}$, Beiträge Algebra Geom. 50 (1) (2009), 235-248.

[15] Radić, M., Sušanj, R., An application of the determinant of a rectangular matrix in discovering some properties of the pentagon, Glas. Mat. Ser. III 27(47) (2) (1992), $217-226$

[16] Radić, M., Sušanj, R., On determinants of rectangular matrices which have Laplace's expansion along rows, Glas. Mat. Ser. III 47(67) (1) (2012), 175-180.

[17] Radić, M., Sušanj, R., Trinajstić, N., Certain classes of polygons in $R^{2}$ and areas of polygons, Rad Hrvat. Akad. Znan. Umjet. Mat. Znan. 16(503) (2009), 7-12.

[18] Stojaković, M., Determinant of non-square matrix, Bull. Soc. Math. Phys. Serbie 4 (1952), 9-23 (Serbo-Croatian).

[19] Sudhir, A. P., On the determinant-like function and the vector determinant, Adv. Appl. Clifford Algebr. 24 (3) (2014), 805-807.

[20] Sušanj, R., Radić, M., Geometrical meaning of one generalization of the determinant of a square matrix, Glas. Mat. Ser. III 29(49) (2) (1994), 217-233.

[21] Yanai, H., Takane, Y., Ishii, H., Nonnegative determinant of a rectangular matrix: Its definition and applications to multivariate analysis, Linear Algebra Appl. 417 (1) (2006), 259-274. 
Anna Makarewicz

Department of Applied Mathematics

Piotr Pikuta

Lublin University of Technology

Institute of Mathematics

ul. Nadbystrzycka 38D

Maria Curie-Skłodowska University

20-618 Lublin

Poland

pl. M. Curie-Skłodowskiej 1

20-031 Lublin

e-mail: a.makarewicz@pollub.pl

Poland

e-mail: ppikuta@poczta.umcs.lublin.pl

Received September 24, 2020 